\title{
Effectiveness and cost efficiency of monitoring mountain nyala in Bale Mountains National Park, Ethiopia
}

\author{
Anouska A. Kinahan ${ }^{1, *}$, Nils Bunnefeld ${ }^{2}$ \\ ${ }^{1}$ Frankfurt Zoological Society, Bale Mountains Conservation Project, Bale Mountains National Park, PO Box 165, Robe, Bale, \\ Ethiopia \\ ${ }^{2}$ Department of Life Sciences, Imperial College London, Silwood Park, Ascot, SL5 7PY, UK
}

\begin{abstract}
Due to the financial limitations faced by many protected areas today, identifying costefficient monitoring protocols has become important in ensuring the long-term sustainability of conservation. The selection of monitoring protocols is usually driven by a range of factors, such as widespread practice or accuracy, but the cost efficiency of protocols is rarely considered. The mountain nyala Tragelaphus buxtoni, classified by the IUCN as Endangered, is endemic to the Ethiopian highlands. This species has high economic potential for local communities through tourism and trophy hunting, but the expansion of human settlement is causing habitat degradation and fragmentation. A significant proportion of the global mountain nyala population occurs in Bale Mountains National Park (BMNP); thus the development of a long-term monitoring protocol was identified as a priority. Like many protected areas, the BMNP is operating well below its financial needs; hence developing a robust, cost-effective method that can detect changes in population size is important. We compared the effectiveness and cost efficiency of distance sampling and total counts. Results showed that while the population estimates were relatively similar, total counts underestimated population size but were more precise, had a greater power to detect changes in population size and required only $12 \%$ of the resources needed compared to distance sampling. We suggest that investing in initial comparisons of the effectiveness and costs of different methods can result in significant cost savings, without jeopardizing the effectiveness of a survey.
\end{abstract}

KEY WORDS: Ecological monitoring $\cdot$ Census techniques $\cdot$ DISTANCE $\cdot$ Protected areas $\cdot$ Total counts

Resale or republication not permitted without written consent of the publisher

\section{INTRODUCTION}

Long-term ecological monitoring is essential for conservation because it provides crucial information on the state of the system and the effectiveness of management interventions (Sinclair et al. 2000, Yoccoz et al. 2001, Singh \& Milner-Gulland 2011). Ecological long-term monitoring reduces uncertainty and thus forms the basis for managers' and stakeholders' decision-making (Bunnefeld et al. 2011) and lays the foundation for adaptive management (Kendall 2001, Nichols \& Williams 2006). As many pro- tected areas already operate with limited funds (Moore et al. 2004), resources for ecological monitoring are often limited. This makes prioritization and cost efficiency of monitoring activities particularly important (Caughlan \& Oakley 2001).

Despite financial limitations, monitoring protocols in most protected areas are typically selected for accuracy and precision (effectiveness), with little attention given to cost effectiveness (efficiency) (GaidetDrapier et al. 2006; for an exception see Hockley et al. 2005). Thus, selecting monitoring methods that maximize ecological effectiveness and economic efficiency 
is a key question for applied ecology, management and conservation (Elphick 2008). Few case studies have assessed the effectiveness of different monitoring protocols based on site-specific pilot studies. For example, in the tall flood plains of India, total counts were more accurate for barasingha Cervus duvauceli, whereas distance sampling (using the programme DISTANCE; Thomas et al. 2012) provided better estimates for species such as wild boar Sus scrofa in the same habitat type (Wegge \& Storaas 2009). Hassel-Finnegan et al. (2008) showed that animal-toobserver distance was more accurate than using the DISTANCE approach for white-handed gibbons $\mathrm{Hy}$ lobates lar carpenteri and Phayre's leaf monkeys Trachypithecus phayrei crepusculus in Thailand. Marshall et al. (2008) reviewed different line-transect methods, including DISTANCE and strip (truncated distance) transects, to estimate primate density and suggested a decision tree based on feasibility of correct estimates of distances and modelling expertise. However, even fewer studies have compared the cost efficiency of different sampling methods.

The mountain nyala Tragelaphus buxtoni is a large ungulate endemic to southern Ethiopia, principally the Bale Mountains, and is listed as Endangered in the IUCN Red List (IUCN 2011). Between $95 \%$ (Evangelista 2006) and $30 \%$ (Atickem et al. 2011) of the population, depending on the source of the data, occurs in Bale Mountains National Park (BMNP). Much of the mountain nyala's habitat has become highly fragmented by human settlement and agriculture. This species is a major tourist attraction for the park, providing alternative livelihood opportunities for 2 park-associated communities. It is also a trophy hunting species. Hunting permit fees for this species are amongst the highest in the country, fetching up to US\$15000 per trophy with 24 permits being sold in 2009/2010 (Ethiopian Wildlife and Conservation Authority pers. comm.). Thus this species has the potential to provide significant economic benefits to local communities. For these reasons, developing a standard long-term monitoring protocol for mountain nyala was identified as a major priority by stakeholders for the BMNP's ecological monitoring programme (Kinahan 2010).

Historically, total counts were used to assess mountain nyala populations in the BMNP. Total counts assume that all individuals are observed, but this assumption is difficult to test for mountain nyala because of dense vegetation in the area. Moreover, total counts do not provide confidence limits, making the precision of estimates difficult to assess. Distance sampling has become an increasingly popular method for population surveys (Plumptre 2000, Focardi et al. 2002, Karanth et al. 2004, Wegge \& Storaas 2009). Given BMNP's limited resources, a robust, cost-efficient method is required if long-term sustainability is to be ensured. Hence, in the present study we compare the effectiveness and cost efficiency of total counts and line transect distance sampling (Buckland et al. 2001) as long-term monitoring methods for assessing mountain nyala population trends in the BMNP.

\section{MATERIALS AND METHODS}

\section{Study area}

BMNP $\left(2200 \mathrm{~km}^{2}\right)$ is located in the south-eastern highlands of Ethiopia (39 $28^{\prime}$ to $39^{\circ} 57^{\prime} \mathrm{E}$ and $6^{\circ} 29^{\prime}$ to $\left.7^{\circ} 10^{\prime} \mathrm{N}\right)$. The park has a large altitudinal range (1500 to $4377 \mathrm{~m}$ above sea level, asl), including the largest expanse of afro-alpine habitat in Africa and the second largest moist tropical forest in Ethiopia. The Gaysay Valley $\left(34.4 \mathrm{~km}^{2}\right)$, which covers $1.6 \%$ of the park's area, is located in the northern part of the park at 3200 to $3500 \mathrm{~m}$ asl (Fig. 1). This area is dominated by open and swampy grassland on chromic luvisol and eutric cambisol soil and is surrounded by Juniper

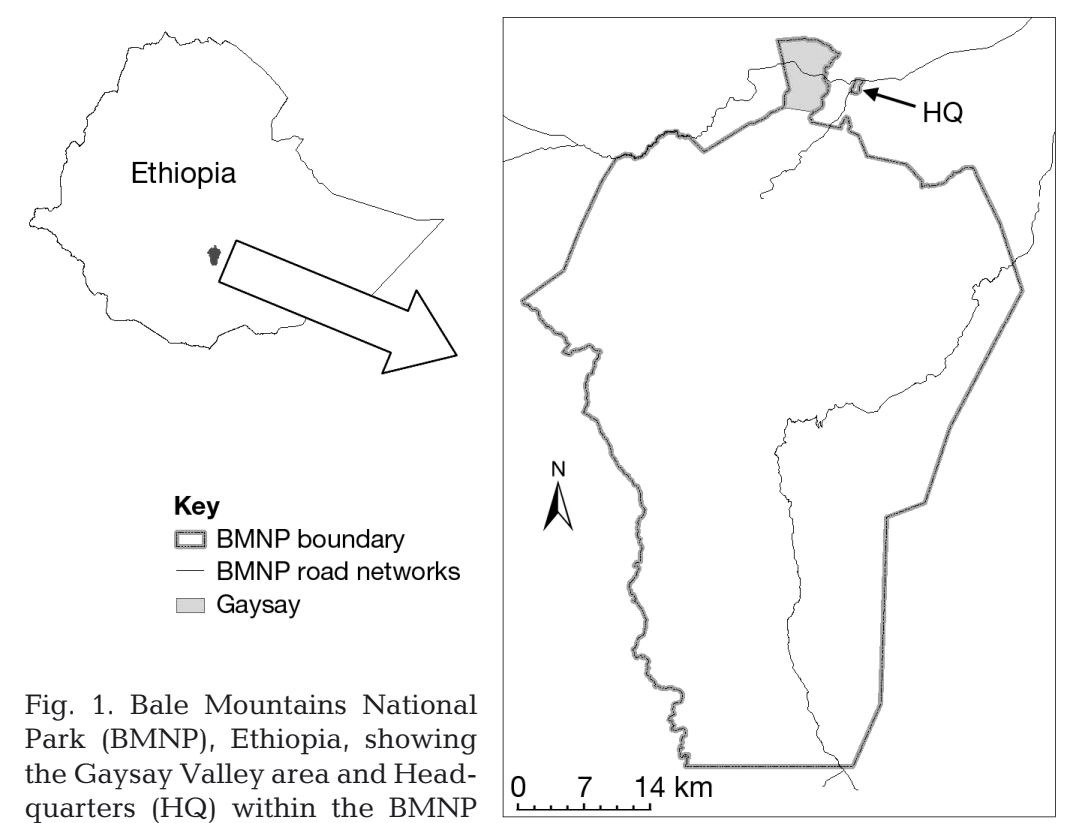


spp. and Hagenia spp. woodland and Erica spp. forest (OARDB 2007). Climate in this area can be divided into the dry season (November to February), the early rains (March to June) and the rainy season (July to October). Average annual rainfall is between 900 and $1300 \mathrm{~mm}$, with average minimum and maximum temperatures of 10 and $12.5^{\circ} \mathrm{C}$ (OARDB 2007). Seventy-seven percent of the park's mountain nyala population (estimated $n=1500$ ) occurs in the Gaysay Valley, with the remaining $22 \%$ at the nearby park Headquarters (see Fig. 1) and $<1 \%$ in other areas of the park (Kinahan 2010).

\section{Sampling techniques}

Total counts

We placed a total of 6 zigzag transects across 10 clearly defined vegetation blocks in the Gaysay Valley (Fig. 2). Zigzags are preferred to parallel lines in cases where resources are limited or counting is expensive (e.g. shipboard and aerial transects; Strindberg \& Buckland 2004, Waltert et al. 2009). Zigzag transects allowed for shorter distances in between transects while covering the desired area to count all individuals, compared to parallel straight line transects with increasing travel distances between transects. The vegetation blocks were chosen by considering the natural changes in vegetation type, for example from shrubland to woodland and where natural features such as rivers occurred. Transects were between 21 and $43 \mathrm{~km}$ in length, with a total transect length of $198 \mathrm{~km}$ traversing the entire Gaysay area. Where transects were longer than $30 \mathrm{~km}$ (4 of 6), we divided the transect in half and 2 different teams walked half of the transect each. We located zigzag points $500 \mathrm{~m}$ apart in open grasslands and shrubland habitat transects (because mountain nyala, with a shoulder height of 120 to $135 \mathrm{~cm}$, are taller than grasses and shrubs), whereas we sampled forested habitats more intensively with zigzag points placed $250 \mathrm{~m}$ apart, since we assumed detection to be more difficult and at shorter distances than for more open habitats. A key assumption of total counts is that all individuals in the covered area are detected.

Teams comprising 2 individuals, a core nyala monitoring team member and a local community member, walked each transect or half transect. The core nyala monitoring team had been trained in GPS use and data recording and participated in annual censuses $(n=4)$. Community members $(n=16)$ acted as spotters and more importantly to promote and encourage community participation in park conservation activities. Members of different Gaysay Valley communities were selected each year in order to try and share benefits more equitably. To avoid double counting, teams were instructed to record individuals only within the designated strata or stratum where their transect lay. All teams commenced each tran-
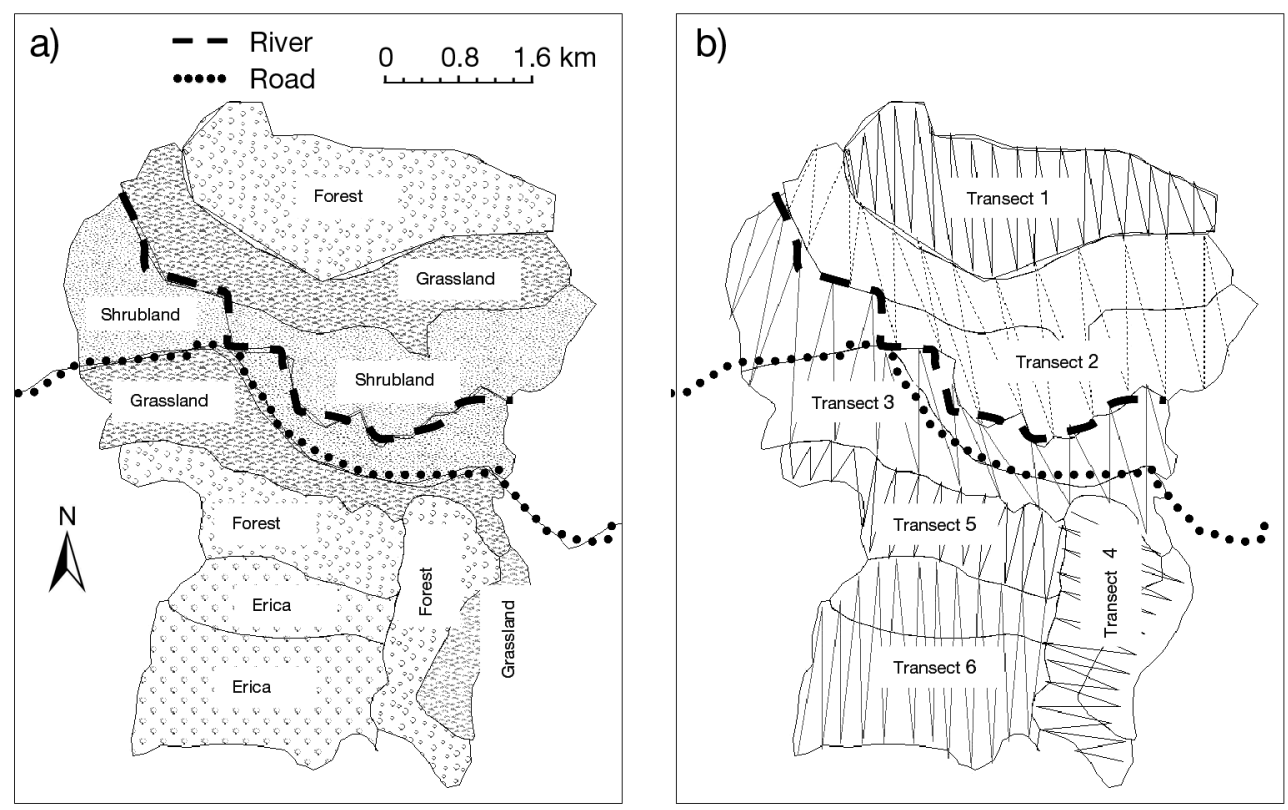

Fig. 2. (a) Ten vegetation blocks identified for the Gaysay Valley (A. Atikem pers. comm.) and (b) the 6 transects that traversed the area across these vegetation blocks that were used to carry out total counts. Each transect was walked once on the same day for total counts, and this protocol was carried out 3 times in 2007 but only once in 2008 and 2010 
sect at the same time $(10: 00 \mathrm{~h})$, and all transects were completed within $1 \mathrm{~d}$. Thus, the entire area was covered at the same time. The number of people involved is presented in Table 1.

We repeated the same sampling procedure 3 times in order to examine the precision of the total counts. We carried out the counts on 3 consecutive days in 2007, to minimize variation due to immigration and emigration of individuals and seasonal movements in and out of the Gaysay Valley. Due to limited financial and personnel resources, total counts were replicated only once in 2008 and 2010, and were not conducted in 2009.

\section{Distance sampling}

We placed 25 transects in the Gaysay Valley across the same geographic area as total counts, following a systematic parallel grid with transects at a $10^{\circ}$ bearing north and south of the road that traverses the area (Fig. 3). We followed the approach of a systematic design using parallel lines with a random first start instead of zigzag lines as outlined by Buckland et al. (2001). Transects were at least $500 \mathrm{~m}$ apart and avoided river crossings. Due to a steep ridge on the northern edge of the Erica spp. habitat zone, which prevents transects from the woodland zone extending into this zone, we placed Erica habitat transects in an east-west $\left(90^{\circ}\right.$ bearing) direction. The 25 transects ranged from 0.65 to $3.5 \mathrm{~km}$ in length and totalled $44.1 \mathrm{~km}$.

Two teams of trained mountain nyala monitors walked 2 different transects per day, resulting in a $6 \mathrm{~d}$ period required to cover all transects. Transects for each team occurred on different sides of the roads, and each team walked a different transect at least $1 \mathrm{~km}$ apart in the morning and afternoon. Otherwise, the order of transects walked was randomly selected during protocol development, but this order remained the same in each year of sampling.

Data were collected using standard line transect distance sampling methods (Buckland et al. 2001), where the distance $(\mathrm{m})$ and angle of each sighting of nyala or group of nyalas (and the total number of individuals) to the transect line were recorded using

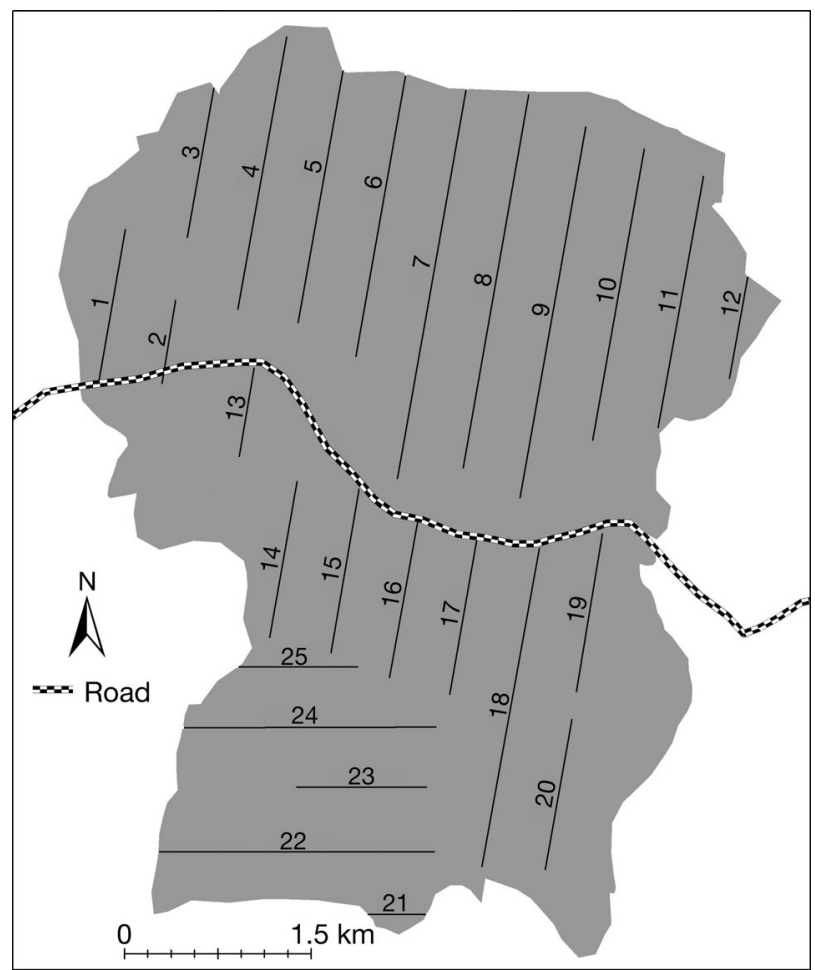

Fig. 3. Locations of the 25 transects across the Gaysay Valley for distance sampling. Each of the 25 transects was walked once over a $6 \mathrm{~d}$ period, and this protocol was repeated 3 times in the same season in 2007 and 2010

Table 1. Breakdown of the total field resources required to implement both methods effectively, and their costs in US dollars. L: total transect length $(\mathrm{km})$

\begin{tabular}{|c|c|c|c|c|c|c|c|}
\hline \multirow[t]{2}{*}{ Resource } & \multirow{2}{*}{$\begin{array}{l}\text { Unit cost } \\
\qquad(\$)\end{array}$} & \multicolumn{3}{|c|}{ Total counts $(\mathrm{L}=198)$} & \multicolumn{3}{|c|}{ Distance $(\mathrm{L}=132.3)$} \\
\hline & & No. of units & No. of days & Total cost $(\$)$ & No. of units & No. of days & Total cost $(\$)$ \\
\hline Para-ecologist salary & 2.70 & 4 & 1 & 10.80 & 4 & 18 & 194.40 \\
\hline $\begin{array}{l}\text { Para-ecologist field } \\
\text { allowance }\end{array}$ & 2.50 & 4 & 1 & 10.00 & 4 & 18 & 180.00 \\
\hline Field assistants & 3.75 & 16 & 1 & 60.00 & 0 & 18 & 0 \\
\hline Camp attendant & 3.50 & 0 & 1 & 0.00 & 1 & 18 & 63.00 \\
\hline Food allowance & 1.50 & 20 & 1 & 30.00 & 5 & 18 & 135.00 \\
\hline Batteries (pack of 4) & 4.00 & 10 & 1 & 40.00 & 8 & 18 & 576.00 \\
\hline Kerosene $\left(1^{-1}\right)$ & 1.00 & 0 & 1 & 0.00 & 18 & 18 & 324.00 \\
\hline Fuel (1-way = 7 km) & 4.00 & 2 & 1 & 8.00 & 4 & 18 & 288.00 \\
\hline Total cost $(\$)$ & & & & 158.80 & & & 1760.4 \\
\hline Cost $\mathrm{km}^{-1}$ & & & & 0.80 & & & 13.32 \\
\hline
\end{tabular}


a manual range finder and a compass. Each sampling procedure was repeated 3 times in 2007 and 2010, but only once in 2008 and 2009 as a result of limited resources. Thus, each of the transects was walked once over a 6 d period (Days 1 to 6 ; Replicate 1), then repeated again in the same order over the next consecutive 6 d (Days 7 to 12, Replicate 2) and then repeated a third time (Days 13 to 18, Replicate 3).

To analyse our data, we used conventional distance sampling with the DISTANCE Software v. 6 for clustered animals (Thomas et al. 2010). To estimate the probability of detection, the uniform, half-normal and hazard rate functions with polynomial or cosine series expansion were fitted. The model with the lowest Akaike Information Criterion (AIC) was selected as best model. Density and encounter rate were estimated from the best model. Detection functions were modelled using exact distances, and to reduce observer bias, the same observers were used throughout the entire protocol in each year. For repeated sampling, the sampling effort was multiplied by the number of times the transects were replicated in each season. In the Discussion, we compare our results against the main assumption of DISTANCE sampling that detection is perfect on the line. We carried out both DISTANCE and total counts in September, during the late wet season, when nyala are most likely to spend their days in open areas rather than woodlands, hence maximizing visibility, and to avoid any seasonal population or detectability differences between the 2 methods.

\section{Measuring effectiveness}

Surveys were considered effective if they could meet the long-term mountain nyala monitoring objective, defined as 'to obtain repeatable mountain nyala population counts in order to detect long term trends in population size' (Kinahan \& Randall 2010, p. 42).

Two indicators were identified as measures of effectiveness:

(1) Precision: repeatability of the survey, where the same or similar population estimates are obtained with repeat sampling under similar conditions. The coefficient of variation in percent (\%CV) between repeated surveys within the same season was used to assess precision. A lower \% CV implied less variation between the estimates and thus a greater confidence in the repeatability of the survey design. The $\% \mathrm{CV}$ between survey estimates was calculated by the following equation:

$$
\% \mathrm{CV}=\frac{S}{x} \times 100
$$

where $s$ is the standard deviation of the population estimates, and $x$ is the mean value across the estimates.

(2) Power to detect change: the likelihood to detect a given percentage change in population size. This was measured using the resolution of a density estimate $(\mathrm{R})$, which is defined as the percentage change that will be detected between 2 independent surveys (Plumptre 2000). Thus, given a resolution of a density estimate of 0.2 (using the CV of the survey estimate), the population would have to increase or decrease by $20 \%$ between 2 independent surveys for the changes to be detectable. Typically, $80 \%$ power to detect change is used (Plumptre 2000).

To determine $80 \%$ power to detect change, the resolution ( $\mathrm{R}$ ) is calculated by

$$
\mathrm{R}=3.96 \mathrm{SE}\left(\frac{\mathrm{D}_{1}}{\mathrm{D}_{2}}\right)=3.96\left(\frac{\mathrm{CV}}{100}\right)
$$

where CV is the coefficient of variation between the samples, $\mathrm{SE}$ is the standard error, and $\mathrm{D}_{1}$ and $\mathrm{D}_{2}$ are the density estimates at time $t$ and $t+1$ (Plumptre 2000).

The population estimates and the number of sightings obtained through DISTANCE and total counts were compared with a Mann-Whitney $U$-test because the data were not normally distributed and the sample size differed for the 2 methods.

\section{Measuring cost efficiency}

The total costs (US\$) required for both methods considering manpower and consumable costs are provided in Table 1. Given the number of individual sightings required for DISTANCE, which is around 100 as a general guideline (Buckland et al. 2001), it required each of the transects to be walked 3 times (Table 2); we used this as our basis for resource calculation for distance sampling. Standard field equipment costs such as GPS, range finders and compass were not considered here as they were required for other monitoring protocols and so were regarded as standard park equipment.

\section{RESULTS}

\section{Effectiveness}

Table 2 shows the results of both survey methods in each of the years sampled. Although total counts 
Table 2. Tragelaphus buxtoni. Comparison of the number of individuals seen in total counts (Tot[N]), the total number of individuals seen in distance sampling (Ds[N]) and the population estimates obtained from DISTANCE analysis (Ds[estimated n]). \% CV is the coefficient of variation in percent of the DISTANCE population estimates and cluster size is the average number of individuals occurring in 1 group or per sighting. The number of groups sighted is given for total (Tot) and distance (Ds) sampling

\begin{tabular}{|c|c|c|c|c|c|c|c|c|c|c|c|}
\hline \multirow[t]{2}{*}{ Year } & \multirow[t]{2}{*}{ Replicate } & \multirow{2}{*}{ Tot $(\mathrm{N})$} & \multirow[b]{2}{*}{ Ds $(\mathrm{N})$} & \multirow{2}{*}{$\begin{array}{l}\text { Population size } \\
\text { Ds (estimated n, } 95 \% \mathrm{CI})\end{array}$} & \multirow[b]{2}{*}{ Ds $(\% \mathrm{CV})$} & \multicolumn{2}{|c|}{ No. of group sightings } & \multicolumn{2}{|c|}{ Encounter rate $\left(\mathrm{km}^{-1}\right)$} & \multicolumn{2}{|c|}{ Cluster size } \\
\hline & & & & & & Tot & Ds & Tot & Ds & Tot & Ds \\
\hline \multirow[t]{4}{*}{2007} & 1 & 774 & 614 & 760 (399-1447) & 33 & 78 & 33 & 0.39 & 0.75 & 7.5 & 7.2 \\
\hline & 2 & 740 & 309 & $2560(1172-5591)$ & 25 & 60 & 31 & 0.30 & 0.70 & 7.3 & 8.2 \\
\hline & 3 & 759 & 510 & $1838(1006-3360)$ & 29 & 54 & 35 & 0.27 & 0.79 & 7.8 & 8.1 \\
\hline & Pooled & & 1433 & 950 (640-1409) & 20 & & 99 & & 0.75 & 7.5 & \\
\hline 2008 & 1 & 848 & 1011 & 1131 (642-1994) & 29 & 40 & 33 & 0.20 & 0.75 & 6.1 & 6 \\
\hline 2009 & 1 & & 500 & $1052(564-1961)$ & 32 & & 31 & & 0.70 & 7.0 & \\
\hline \multirow[t]{4}{*}{2010} & 1 & 1280 & 421 & $1208(737-1664)$ & 20 & 120 & 56 & 0.61 & 1.27 & 5.3 & 5.6 \\
\hline & 2 & & 333 & 1645 (953-2839) & 28 & & 42 & & 0.95 & 6.8 & \\
\hline & 3 & & 320 & $1697(1043-2760)$ & 25 & & 54 & & 1.22 & 5.9 & \\
\hline & Pooled & & 1074 & 1463 (1087-1968) & 15 & & 152 & & 1.15 & 5.9 & \\
\hline
\end{tabular}

continuously provided lower population estimates compared to DISTANCE, all total counts fell within the confidence intervals provided by the pooled DISTANCE analysis, and no significant difference in the population estimates were observed (Mann-Whitney $U=3, \mathrm{p}>0.1)$. Total counts showed an average annual population increase of $20 \%$ compared to $17 \%$ from DISTANCE.

Generally, DISTANCE provided significantly lower numbers of sightings per survey compared to total counts (Mann-Whitney $U=4.5, \mathrm{p}<0.03$ ). On average, 39 (range: 31-56) mountain nyala groups were sighted using 1 replicate of the distance sampling transects. This is well below the recommended minimum number of sightings (100) required for clustered animals to be able to obtain reliable density estimates in DISTANCE analysis (Buckland et al. 2001). Further analysis showed that walking each of the transects 3 times per survey period resulted in near to or more than the recommended 100 sightings ( $\mathrm{N}=99$ in 2007 and 155 in 2010) and thus a more acceptable $\% \mathrm{CV}(\leq 20 \%)$ of population estimates (Table 2).

Fig. 4 shows the detection functions from DISTANCE in each year. Generally, the models show that the detection probability of seeing an animal on the line is 1 , with a decline in detection probability as the animal is farther from the transect. The figure shows that on average the probability of detecting an animal at $\leq 50 \mathrm{~m}$ is $100 \%$, at $\leq 100 \mathrm{~m}$ it is $50 \%$, and at distances $>200 \mathrm{~m}$ there is $<10 \%$ detection probability.

The Gaysay Valley comprises both open and closed habitat types. Due to the small number of sightings possible for each type, we were unable to obtain density estimates by stratifying habitats.
However, the detection functions for both open and closed habitat types shows that the detection probability is $<1$ on or close to the line in closed habitats and, compared to open habitats, has a lower probability of detecting species at a given distance from the line (Fig. 5). Thus, while our assumptions for total counts were true in that the detection probability will be lower in closed compared to open habitats, the detection functions suggest that our $250 \mathrm{~m}$ and $500 \mathrm{~m}$ distance between zigzags overestimated the distance we could actually locate mountain nyala reliably. In closed habitats, the average distance between the 2 zigzag transects was $125 \mathrm{~m}$ (distance ranges between 0 and $250 \mathrm{~m}$, Fig. 2), which gives an average sighting distance of $62.5 \mathrm{~m}$ at any given point on an individual transect (i.e. half the area between 2 transects). Similarly, for open habitats, the average sighting distance expected is $125 \mathrm{~m}$. The detection functions showed that at these distances, after $50 \mathrm{~m}$, there is approximately a $20 \%$ probability of detecting animals. This implies therefore that the total count survey is missing some individuals at distances over $50 \mathrm{~m}$ from the transect line and therefore is likely to provide an underestimate of true population size.

Total counts had a higher repeatability with $2 \%$ CV among the 3 replicates in 2007, whereas DISTANCE sampling showed a wide variation among the 3 replicates, 56 and $18 \%$ in 2007 and 2010, respectively. Total counts $(2 \% \mathrm{CV})$ resulted in $\mathrm{R}=$ 0.07 or an $80 \%$ probability of being able to detect a $7 \%$ change in population size between 2 independent samples. DISTANCE generated a $56 \% \mathrm{CV}$ (R = $2.2)$ in 2007 and $18 \% \mathrm{CV}(\mathrm{R}=0.71)$ in 2010 and hence requires a large change in the population between surveys before it can be detected ( 22 and $71 \%$ ). 

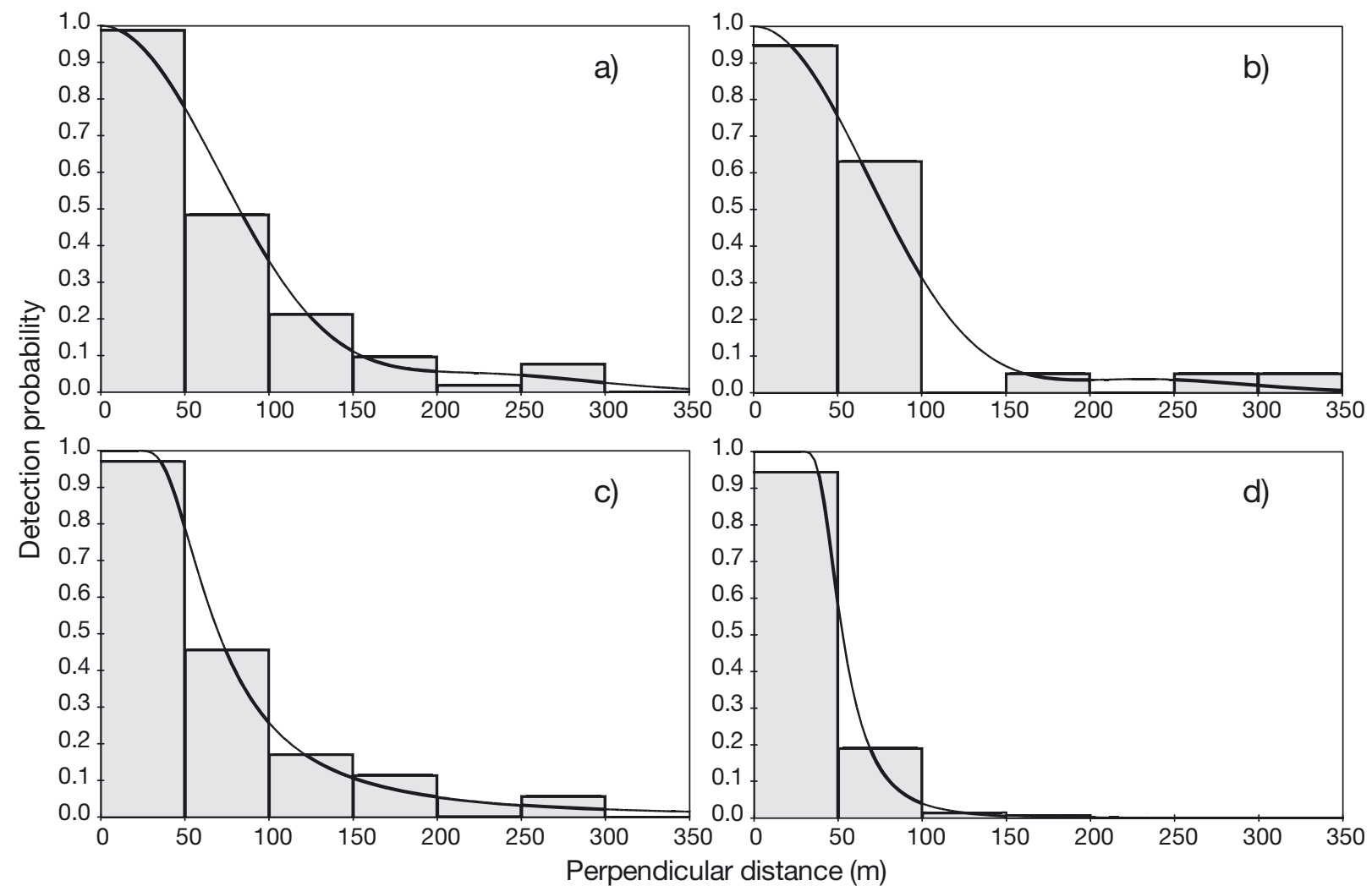

Fig. 4. Detection functions for (a) 2007, (b) 2008, (c) 2009 and (d) 2010; for all years the hazard rate model (smooth line) had the best fit. The models show that the detection probability of seeing an animal on the transect line is 1 , with a decline in detection probability with increasing distance of the animal from the transect

\section{Efficiency}

Table 2 shows that for effort, distance sampling is the most efficient survey method, with greater encounter rates per $\mathrm{km}$ transect walked $\left(0.90 \pm 0.23 \mathrm{~km}^{-1}\right)$ compared to total counts $\left(0.36 \pm 0.13 \mathrm{~km}^{-1}\right)$. However, the cost for walking $1 \mathrm{~km}$ of transect is almost 16 times lower for total counts than for distance sampling (Table 1). The main cost increase was due to the fact that sampling effort needed to be increased from $1 \mathrm{~d}$ for the total counts to $6 \mathrm{~d}$ repeated 3 times (= $18 \mathrm{~d}$ ) for distance sampling. The untrained field assistants required for total counts were not needed for the distance method because all field workers needed to be trained. However, this resulted only in small savings for distance sampling compared to total counts (Table 1). The higher number of days out in the field for distance sampling increased the costs of the salary and the field allowance for para-ecologists, the food allowance, batteries and fuel and added costs for kerosene and a camp attendant for the period of the field work. Thus, although distance sampling may be more than twice as efficient in terms of encounter rate, the cost is 16 times more than for total counts.

\section{DISCUSSION}

We show that considering effectiveness as well as cost efficiency, a factor not often explicitly incorporated into monitoring protocol choice, can result in significant cost saving in the long term. Although distance sampling is typically considered a preferable survey method (Buckland et al. 2001, Karanth et al. 2004), in some circumstances, such as in the case of monitoring the mountain nyala population in Gaysay, it may not be the most effective or cost-efficient method to meet monitoring objectives.

\section{Population size}

Our results showed that the number of mountain nyala occurring in Gaysay Valley increased between 2007 and 2010 from 774 to 1280 individuals using total counts and from 950 to 1463 individuals using DISTANCE. However, $95 \%$ confidence intervals overlapped for the results estimated by the DISTANCE method (Table 2), indicating that given the variation, the increase in the population size is not 


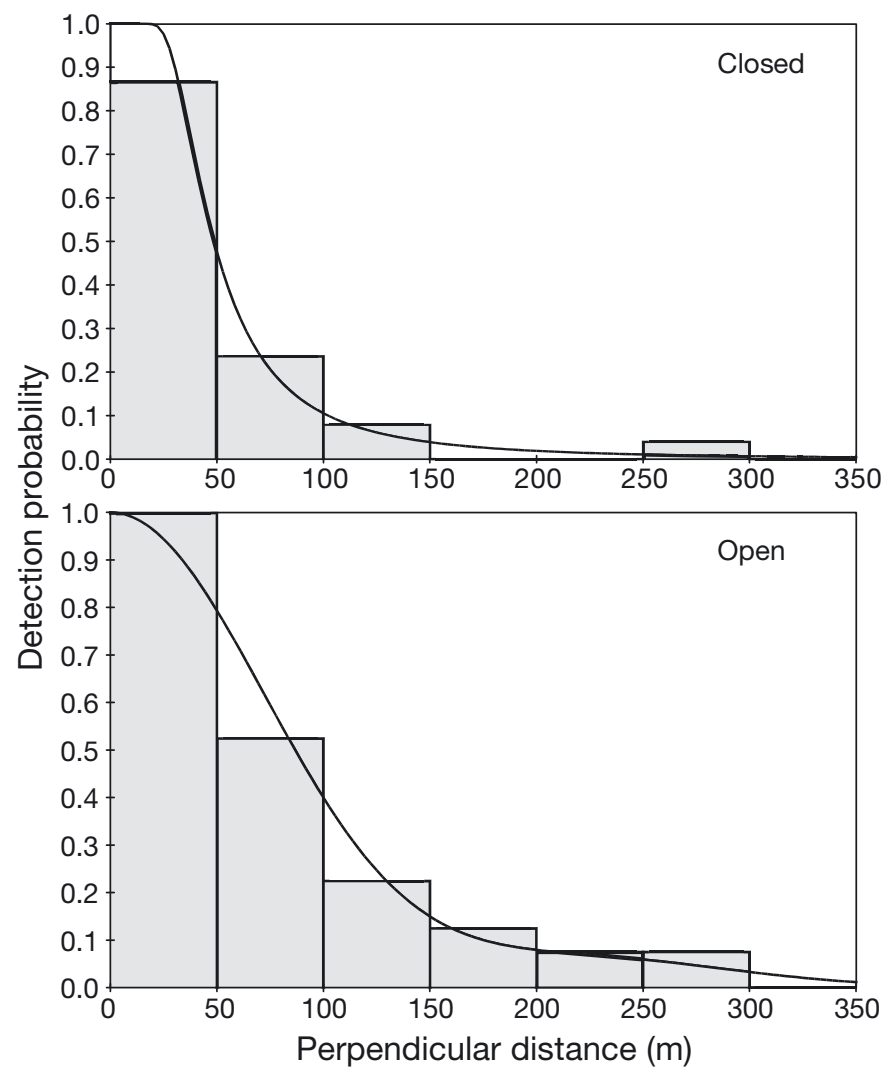

Fig. 5. Detection functions in closed and open habitats for mountain nyala in the Gaysay Valley. Smooth lines: best fit using the hazard rate model

strongly supported due to large variation in the data. Atickem et al. (2011) used faecal samples to estimate the population size of the mountain nyala to be 776 individuals (95\% CI: 528-1290) in the Gaysay Valley, which is only 2 individuals more than our results obtained from the total counts. The 3 replicates from our total counts in 2007 yielded a small variation $(2 \% \mathrm{CV})$ between counts. DISTANCE detection functions suggested that our total counts did not detect all individuals on the line as previously assumed, and that despite our efforts to mitigate the variability in sighting distances (by reducing the distance between zigzags in more dense vegetation), our total counts likely still underestimated the population size. This is in line with a review by Gaillard et al. (2003), who showed that total counts generally underestimate population size by 22 to $62 \%$, particularly in habitats where visibility is very limited, e.g. dense forests (Houssin et al. 1994, Cano et al. 2009). However, total counts provided values that fell within the confidence limits of the pooled DISTANCE analysis, and both methods yielded similar cluster size values.

\section{Precision and power to detect change}

For conservation managers, population trends, rather than absolute levels, are an important determinant for management evaluation and intervention. In this study, total counts of mountain nyala were both more precise and exhibited greater power to detect change than distance sampling. DISTANCE estimates had overlapping confidence limits over the years, lower precision between independent surveys (CVs of 56 and $18 \%$ ) compared to total counts (CV of $2 \%$ ) and lower power to detect changes in population size than total counts. This suggests that total counts provide better information on population trends and thus are more reliable for decisions on management interventions. Similar to Wegge \& Storaas (2009), we found that DISTANCE confidence limits are linked to the number of sightings, with low sighting numbers resulting in wide confidence intervals, and confidence intervals becoming narrower as the number of sightings increased (Table 2). Thus, in DISTANCE, it is harder to detect declining trends in small populations, since confidence intervals increase as population size decreases, and so confidence intervals are more likely to overlap.

\section{Cost efficiency}

This study showed that cost efficiency was much higher for total counts, with 20 times lower cost per survey compared to distance sampling. Although we sustained a high initial investment to compare these monitoring methods, US $\$ 1169$ per annum, or US $\$ 11696$ over the next $10 \mathrm{yr}$, can now be saved by using the more cost-efficient method. This is a significant savings, representing $5 \%$ of the current annual funds available to the park for their ecological monitoring programme (Kinahan 2011).

\section{Total counts versus DISTANCE}

Many technical monitoring schemes such as distance sampling require considerable funds and scientific expertise and produce results which are generally not as quick and easy to interpret or act upon as simpler methods such as total counts (Danielsen et al. 2010). In this study, we have not included the scientific expertise and the number of hours that were used by experienced quantitative scientists to analyse DISTANCE sampling data because it is hard to estimate the cost of having a quantitative scientist 
available for a single study. This means that we have calculated only the minimum costs in this study; the real cost of distance sampling might be considerably higher. For mountain nyalas in BMNP, total counts are transparent to the local people, provide a greater economic benefit to the communities through temporary employment and involve more community members in park activities than DISTANCE sampling. As a result, local communities can relate directly to field observations and results are immediately transparent, whereas DISTANCE involves significant time for data entry and expertise in its analysis and interpretation. Total counts are therefore more likely to be accepted in the local community, generate community support and improve protected area-community relations (Danielsen et al. 2005). There is increasing evidence that stakeholder involvement and acceptance is crucial for conservation success (Waylen et al. 2010, Bunnefeld et al. 2011), which can be further promoted by including local people in monitoring activities such as total counts.

Our results show that total counts were more effective (in meeting monitoring objectives) and cost efficient than distance sampling in the Gaysay Valley of the BMNP. However, it should be noted that the best monitoring method depends, among other factors, on the scale, the habitat, the biology of the species and the budget of the case study (Singh \& Milner-Gulland 2011). Previous studies have shown that while total counts may be useful for rare or elusive species (Wegge \& Storaas 2009), or species not easily detected on the line (Rodda \& Campbell 2002, Smolensky \& Fitgerald 2010), distance sampling may be more preferred for species that are large and easily detected (Focardi et al. 2005, Durant et al. 2011), where individuals are numerous (Wegge \& Storaas 2009), where there is dense vegetation (Herrero et al. 2011) or where large areas not easily covered by total counts occur. The suitability of total counts is perhaps not surprising in the Gaysay Valley, given its small area $\left(34 \mathrm{~km}^{2}\right)$, low coverage of dense forest and the small number of mountain nyalas. However, a large disadvantage is the lack of confidence limits surrounding the total count estimates and the uncertainty as to how detection levels change with changes in population size and habitat types. Hence, given the cost savings of total counts, where possible total counts should be repeated more often than once a year, and resources permitting, distance sampling should be carried out every 3 to $5 \mathrm{yr}$ in order to attain confidence limits around the estimates and to allow for differences in detectability due to changing population size and habitat.

\section{CONCLUSION}

Total counts are more effective and cost efficient in achieving the monitoring objective of repeatable population counts in order to detect long-term trends in population size for mountain nyala in Gaysay in the BMNP. We showed that the power to detect a change in population size was high, with an $80 \%$ probability of being able to detect a $7 \%$ change in the population. Our study demonstrated that the initial high financial investment in comparing both the effectiveness and cost efficiency of 2 common population survey methods revealed important insights for the future monitoring scheme of mountain nyala, and will result in dramatic cost savings over the long term. Furthermore, total counts allow for increased involvement of local people and higher transparency of the data-to-results process among stakeholders and managers. Local community and stakeholder involvement is of key importance for conservation success and is more likely to be achieved through total counts than through distance sampling. In general, initial investment into a pilot study comparing methods is worth the cost in the long term and is a practice that should be adopted for monitoring programmes in other protected areas. We highlight the importance of including economic costs when comparing monitoring methods in pilot studies so that appropriate recommendations can be provided for monitoring plans that are economically feasible in the long term.

Acknowledgements. We thank D. Randall and A. Atickem for their input into the distance sampling and total count protocol development. We also thank S. Newey for helpful comments on the distance sampling protocol and the final draft of this manuscript. Thanks to J. Jones, N. Hockley, A. Keane, J. Fa and K. Laurenson for helpful comments on various stages of this manuscript. The comments of 2 anonymous referees greatly improved the manuscript. N.B. was supported by the European Commission under the HUNT project of the 7th Framework Programme for Research and Technological Development. Data collection was funded by the Frankfurt Zoological Society and the European Commission. Neither the European Commission nor any person acting on behalf of the Commission is responsible for the use made of the information. The views expressed in this publication are the sole responsibility of the authors and do not necessarily reflect the views of the European Commission.

\section{LITERATURE CITED}

Atickem A, Loe LE, Langangen Ø, Rueness EK, Bekele A, Stenseth NC (2011) Estimating population size and habitat suitability for mountain nyala in areas with different protection status. Anim Conserv 14:409-418

Buckland ST, Anderson DR, Burnham KP, Laake JL, Borchers DL, Thomas L (2001) Introduction to distance 
sampling: estimating abundance of biological populations. Oxford University Press, Oxford

Bunnefeld N, Hoshino E, Milner-Gulland EJ (2011) Management strategy evaluation: a powerful tool for conservation? Trends Ecol Evol 26:441-447

Cano M, García-Rovés P, González-Quirós P, Nores C (2009) Experiencias de estimación de poblaciones de rebeco en la Cordillera Cantábrica. In: Pérez-Barbería FJ, Palacios B (eds) El rebeco cantábrico Rupicapra pyrenaica parva. Conservación y gestión de sus poblaciones. Naturaleza y Parques Nacionales. Serie Técnica. Ministerio de Medio Ambiente, Medio Rural y Marino, Madrid, p 416-447

> Caughlan L, Oakley KL (2001) Cost considerations for longterm ecological monitoring. Ecol Indic 1:123-134

> Danielsen F, Jensen AE, Alviola PA, Balete DS and others (2005) Does monitoring matter? A quantitative assessment of management decisions from locally-based monitoring of protected areas. Biodivers Conserv 14:2633-2652

> Danielsen F, Burgess ND, Jensen PM, Pirhofer-Watzl K (2010) Environmental monitoring: the scale and speed of implementation varies according to the degree of people's involvement. J Appl Ecol 47:1166-1168

> Durant SM, Craft ME, Hilborn R, Bashir S, Hando J, Thomas $\mathrm{L}$ (2011) Long-terms trends in carnivore abundance using distance sampling in Serengeti National Park, Tanzania. J Appl Ecol 48:1490-1500

> Elphick CS (2008) How you count counts: the importance of methods research in applied ecology. J Appl Ecol 45: 1313-1320

Evangelista P (2006) The range and distribution of mountain nyala. Report to the Ethiopian Wildlife Department, Addis Ababa

> Focardi S, Isotti R, Tinelli A (2002) Line transect estimates of ungulate populations in a Mediterranean forest. J Wildl Manag 66:48-58

- Focardi S, Montanaro P, Isotti R, Scacco M, Calmanti R (2005) Distance sampling effectively monitored a declining population of Italian roe deer Capreolus capreolus italicus. Oryx 39:421-428

Gaidet-Drapier N, Fritz H, Bourgarel M, Pierre-Cyril R and others (2006) Cost and efficiency of large mammal census techniques: comparison of methods for a participatory approach in a communal area, Zimbabwe. Biodivers Conserv 15:735-754

Gaillard JM, Loison A, Toigo C (2003) Variation in life history traits and realistic population models for wildlife management. In: Festa-Bianchet M, Apollonio M (eds) Animal behavior and wildlife conservation. Island Press, Washington, DC, p 115-132

- Hassel-Finnegan HM, Borries C, Larney E, Umponjan M, Koenig A (2008) How reliable are density estimates for diurnal primates? Int J Primatol 29:1175-1187

> Herrero J, Garcia-Serrano A, Prada C, Fernandez-Arbera O (2011) Using block counts to estimate populations of chamois. Pirineos 166:123-133

> Hockley NJ, Jones JPG, Andriahajaina FB, Manica A, Ranambitsoa EH, Randriamboahary JA (2005) When should communities and conservationists monitor exploited resources? Biodivers Conserv 14:2795-2806

Houssin H, Loison A, Jullien JM, Gaillard JM (1994) Validité de la méthode du pointage-flash pour l'estimation des effectifs de chamois (Rupicapra rupicapra). Gibier Faune Sauvage 11:287-298

IUCN (International Union for Conservation of Nature and Natural Resources) (2011) IUCN Red List of threatened

Editorial responsibility: Dave Hodgson,

University of Exeter, Cornwall Campus, UK species. Version 2011.2. Available at www.iucnredlist.org (accessed on 25 January 2012)

Karanth KU, Nichols JD, Kumar NS, Link WA, Hines JE (2004) Tigers and their prey: predicting carnivore densities from prey abundance. Proc Natl Acad Sci USA 101: 4854-4858

Kendall WL (2001) Using models to facilitate complex decisions. In: Shenk TM, Franklin AB (eds) Modeling in natural resource management. Island Press, Washington, DC, p 147-170

Kinahan AA (2010) An introduction to BMNP's long term monitoring programme. Vol 1. FZS/BMNP Publication, Addis Ababa

Kinahan AA (2011) Bale Mountains National Park business and sustainable financing plan. FZS/BMNP Publication, Addis Ababa

Kinahan AA, Randall DR (2010) Mountain Nyala monitoring protocol. Vol 3c. BMNP Ecological Monitoring Programme. FZS/BMNP Publication, Addis Ababa

Marshall AR, Lovett JC, White PCL (2008) Selection of linetransect methods for estimating the density of groupliving animals: lessons from the primates. Am J Primatol 70:452-462

Moore J, Balmford A, Allnutt T, Burgess N (2004) Integrating costs into conservation planning across Africa. Biol Conserv 117:343-350

Nichols JD, Williams BK (2006) Monitoring for conservation. Trends Ecol Evol 21:668-673

OARDB (Oromia Agriculture and Rural Development Bureau) (2007) Bale Mountains National Park general management plan 2007-17. OARDB, FZS and IBCCSMPP compilation, Addis Ababa

> Plumptre AJ (2000) Monitoring mammal populations with line transect techniques in African forests. J Appl Ecol 37:356-368

Rodda GH, Campbell EW (2002) Distance sampling of forest snakes and lizards. Herpetol Rev 33:271-274

> Sinclair ARE, Ludwig D, Clark CW (2000) Conservation in the real world. Science 289:1875

Singh N, Milner-Gulland EJ (2011) Monitoring ungulates in Central Asia: current constraints and future potential. Oryx 45:38-49

> Smolensky NL, Fitzgerald LA (2010) Distance sampling underestimates population densities of dune-dwelling lizards. J Herpetol 44:372-381

Strindberg S, Buckland ST (2004) Zigzag survey designs in line transect sampling. J Agric Biol Environ Stat 9: 443-461

Thomas L, Buckland ST, Rexstad EA, Laake JL and others (2010) Distance software: design and analysis of distance sampling surveys for estimating population size. J Appl Ecol 47:5-14

> Waltert M, Chuwa M, Kiffner C (2009) An assessment of the puku (Kobus vardonii Livingstone 1857) population at Lake Rukwa, Tanzania. Afr J Ecol 47:688-692

> Waylen K, Fischer A, McGowan P, Thirgood S, MilnerGulland EJ (2010) The effect of local cultural context on the success of community-based conservation interventions. Conserv Biol 24:1119-1129

> Wegge P, Storaas T (2009) Sampling tiger ungulate prey by the DISTANCE method: lessons learned in Bardia National Park, Nepal. Anim Conserv 12:78-84

> Yoccoz NG, Nichols JD, Boulinier T (2001) Monitoring of biological diversity in space and time. Trends Ecol Evol $16: 446-453$

Submitted: February 10, 2012; Accepted: May 10, 2012

Proofs received from author(s): July 26, 2012 